\title{
Self-assembled rosette nanotubes encapsulate and slowly release dexamethasone
}

This article was published in the following Dove Press journal:

International Journal of Nanomedicine

17 May 201 I

Number of times this article has been viewed

\author{
Yupeng Chen ${ }^{1,2}$ \\ Shang Song ${ }^{2}$ \\ Zhimin Yan ${ }^{3}$ \\ Hicham Fenniri ${ }^{3}$ \\ Thomas J Webster ${ }^{2,4}$ \\ 'Department of Chemistry, \\ Brown University, Providence, \\ RI, USA; ${ }^{2}$ School of Engineering, \\ Brown University, Providence, \\ RI, USA; ${ }^{3}$ National Institute for \\ Nanotechnology and Department \\ of Chemistry, University of Alberta, \\ Edmonton, Alberta, Canada; \\ ${ }^{4}$ Department of Orthopedics, Brown \\ University, Providence, RI, USA
}

\begin{abstract}
Rosette nanotubes (RNTs) are novel, self-assembled, biomimetic, synthetic drug delivery materials suitable for numerous medical applications. Because of their amphiphilic character and hollow architecture, RNTs can be used to encapsulate and deliver hydrophobic drugs otherwise difficult to deliver in biological systems. Another advantage of using RNTs for drug delivery is their biocompatibility, low cytotoxicity, and their ability to engender a favorable, biologically-inspired environment for cell adhesion and growth. In this study, a method to incorporate dexamethasone (DEX, an inflammatory and a bone growth promoting steroid) into RNTs was developed. The drug-loaded RNTs were characterized using diffusion ordered nuclear magnetic resonance spectroscopy (DOSY NMR) and UV-Vis spectroscopy. Results showed for the first time that DEX can be easily and quickly encapsulated into RNTs and released to promote osteoblast (bone-forming cell) functions over long periods of time. As a result, RNTs are presented as a novel material for the targeted delivery of hydrophobic drugs otherwise difficult to deliver.
\end{abstract}

Keywords: nanotubes, drug delivery, self-assembly, physiological conditions

\section{Introduction}

Since the first hip replacement surgery in 1923, orthopedic implants have been routinely inserted, increasing the quality of life for millions of people. ${ }^{1}$ A variety of materials have been investigated as bone replacements, such as conventional titanium and its alloys, micron grain sized ceramics (eg, hydroxylapatite or HA) and nanostructured hydrogels (eg, poly-lactic-co-glycolic acid or PLGA). ${ }^{2-5}$ However, current conventional implants suffer from limited lifetimes, often failing before the end of a patient's natural life expectancy. ${ }^{6,7}$ For example, today's orthopedic implants usually have insufficient initial bone growth on their surface and/or poor maintenance of healthy juxtaposed bone over long periods of time. Such problems caused by poor osteointegration of the implanted device can lead to bone loss, implant loosening, and ultimately implant failure. For example, from October 2005 to December 2006, the number of total hip replacement revisions performed in the US reached 51,345 as a result of instability/ dislocation, mechanical loosening and osteolysis. ${ }^{8}$

To enhance the integration of orthopedic implants with bone forming cells (osteoblasts) and surrounding bone, implants with nanostructured surfaces have received a tremendous amount of recent attention. ${ }^{9,10}$ An important member of this class of nanomaterials are the rosette nanotubes (RNTs, Figure 1). ${ }^{11-13}$ RNTs are novel, biomimetic, self-assembled, supramolecular structures, whose basic building blocks feature the hydrogen bonding arrays of guanine $(\mathrm{G})$ and cytosine $(\mathrm{C})$ DNA bases. 


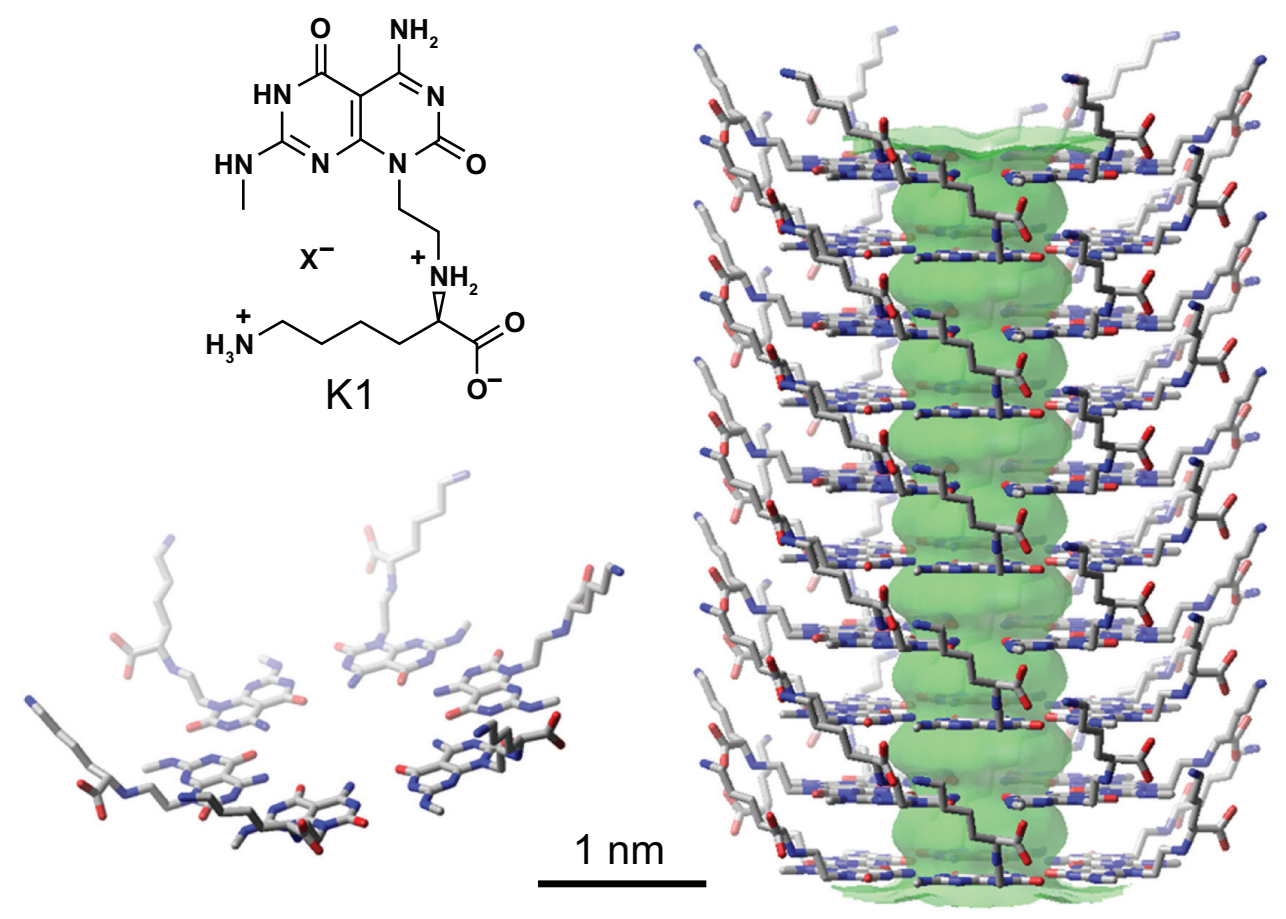

Figure I Schematic illustration of the hierarchical self-assembly of RNTs with a lysine side chain. The single unit of $K$ I (G^C motif conjugated to a lysine amino acid) self-assembles into a rosette ring (lower left), which stacks up to form a stable RNT (right), referred to as KI in this report.

Abbreviation: RNT, rosette nanontubes.

RNTs are produced synthetically ${ }^{13}$ and are assembled under physiological conditions. They have been shown to have low toxicity ${ }^{14,15}$ (in vitro and in vivo) and have been shown to have anti-cancer properties when functionalized with cell adhesive peptides such as arginine-glycine-aspartic acid-serine-lysine (RGDSK). ${ }^{16}$

A heteroaromatic bicyclic base functionalized with L-lysine $(\mathrm{K}-\mathrm{G} \wedge \mathrm{C}$, Figure 1) undergoes a hierarchical self-assembly process under physiological conditions to form a six-membered supermacrocycle maintained by 18 hydrogen bonds. Because of electrostatic forces, base stacking interactions and hydrophobic effects, the rosettes form a stable stack with an $11 \AA$ open channel. L-lysine was chosen to impart chirality, stability, biocompatibility, and water solubility. Thus, each of the RNT building blocks has a lysine chain, and the resulting RNTs (referred to as K1) are covered with lysine amino acids (Figure 1). The biomimetic, nanostructured nature of RNTs provide a favorable environment for cell adhesion and growth. ${ }^{11,12,17,18}$

Due to their synthetic accessibility, RNTs have unique advantages for drug delivery applications because: a) they can be chemically conjugated with bioactive peptides such as RGDSK-RNT (RNTs functionalized with Arg-Gly-Asp-Ser-Lys) to improve osteoblast functions on hydrogels ${ }^{18}$ and b) they can be loaded with drugs via hydrophobic or stacking interactions. ${ }^{19}$

As a first step toward the application of RNTs as novel drug delivery devices for orthopedic applications, we investigated the incorporation of dexamethasone (DEX) into RNTs and characterized the drug-loaded RNTs by diffusion ordered nuclear magnetic resonance spectroscopy (DOSY NMR), atomic force microscopy (AFM), and UV-Vis spectroscopy. DEX is known to enhance osteoconductivity and is widely used in bone tissue engineering. ${ }^{20-22}$ However, this drug interferes with the differentiation of bone marrow-derived stem cells into muscle, fat, cartilage and other tissue-forming cells. ${ }^{23}$ Therefore, it is important to deliver DEX to the desired site of new bone growth to ensure maximum therapeutic activity and specificity. ${ }^{19}$

Results of this study showed that DEX was indeed incorporated into RNTs by simply allowing RNTs to selfassemble in solution in the presence of DEX. Subsequent cell studies demonstrated that DEX was released from the RNTs for up to 9 days and had a positive impact on osteoblast functions compared to DEX added to cell culture media alone. This study thus presents a novel drug delivery system where not only is the delivery device itself bioactive, but it can also deliver a variety of drugs for various medical applications. 


\section{Materials and methods \\ Preparation of RNTs}

The self-assembling $\mathrm{K}-\mathrm{G} \wedge \mathrm{C}$ modules were synthesized according to a previously reported strategy. ${ }^{13}$ They were then assembled into RNTs in distilled water $\left(\mathrm{dH}_{2} \mathrm{O}\right)$ to a final concentration of $100 \mu \mathrm{g} / \mathrm{mL}$.

\section{' $\mathrm{H}$ and DOSY NMR studies}

To test the ability of $\mathrm{K} 1$ to deliver hydrophobic drugs, $4.07 \mathrm{mg}$ of $\mathrm{K}-\mathrm{G} \wedge \mathrm{C}$ were dissolved in deuterated methanol $\left(\mathrm{CD}_{3} \mathrm{OD}, 1 \mathrm{~mL}\right)$ and aged for 1 day at room temperature. Tertbutanol ( $t$-BuOH, $1.3 \mu \mathrm{L}$, [Sigma, St Louis, MO] $\geq 99.5 \%$ anhydrous) was added as a ${ }^{1} \mathrm{H}$ NMR internal standard to quantify the extent of DEX interaction with K1. DEX (5.11 mg, Sigma, D4902) was then added resulting in a solution composed of DEX:K1 at a molar ratio of 5:3. The solution was aged for 2 days prior to ${ }^{1} \mathrm{H}$ NMR and DOSY NMR investigations. Proton NMR spectra and DOSY were recorded on a Varian Direct Drive VNMRS 600 spectrometer (Agilent Technologies, Santa Clara CA) operating at a magnetic field strength of $14.1 \mathrm{~T}\left(600 \mathrm{MHz}{ }^{1} \mathrm{H}\right.$ frequency). A dual broadband probe was used. ${ }^{1} \mathrm{H}$ NMR spectra were acquired at room temperature using a single pulse excitation with a $45^{\circ}$ flip angle of $3.6 \mu$ seconds and an acquisition time of 1.7 seconds. The repetition time was 1.0 seconds. It should be noted that as DEX interacts with the RNTs, the corresponding proton integration and diffusion coefficient decrease proportionally as a result of the large relaxation time of the self-assembled RNTs.

\section{Atomic force microscopy (AFM)}

AFM experiments were carried out on a Digital Instruments/ Veeco Instruments MultiMode Nanoscope IV AFM (Plainview, NY) equipped with an E scanner. Silicon cantilevers (MikroMasch USA, Inc., San Jose, CA) with low spring constants of $4.5 \mathrm{~N} / \mathrm{m}$ were used in tapping mode with a scan rate of $0.5-1 \mathrm{~Hz}$ and amplitude setpoint of $1 \mathrm{~V}$. K1 $(20 \mu \mathrm{L}$ of a $50-250 \mu \mathrm{g} / \mathrm{mL}$ solution) with/without DEX were spincoated on freshly cleaved mica at 2,000 rpm for 20 seconds and dried in air prior to imaging.

\section{UV-Vis spectroscopy}

For UV-Vis experiments (Agilent 8453, Agilent Technologies, Santa Clara, CA), the DEX:K1 (5:3 molar ratio) stock solution containing $4.07 \mathrm{mg}$ of the drug-loaded RNTs in methanol (see above) was diluted to $25 \mu \mathrm{g} / \mathrm{mL}$ with methanol. Solutions of DEX $(5 \mu \mathrm{g} / \mathrm{mL})$ and $\mathrm{K} 1(25 \mu \mathrm{g} / \mathrm{mL})$ in methanol were prepared, as well as the same three solutions in $\mathrm{dH}_{2} \mathrm{O}$.

\section{DEX loading and release studies}

Glass coverslips (Fisher Scientific, Waltham, MA: circular; diameter, $18 \mathrm{~mm}$; thickness, $1 \mathrm{~mm}$ ) were cleaned with methanol, acetone and water in a sonicator. Three groups were prepared in $\mathrm{dH}_{2} \mathrm{O}$ : a) glass coverslips were dipped in a water-soluble DEX solution (1 mg/mL, Sigma, St Louis, MO, D2915); b) glass coverslips were first soaked in a K1 solution $(100 \mu \mathrm{g} / \mathrm{mL})$ and after air-drying, they were then immersed into the water-soluble DEX solution $(1 \mathrm{mg} / \mathrm{mL})$; and c) glass slides were dipped in a $\mathrm{K} 1$ solution $(100 \mu \mathrm{g} / \mathrm{mL})$ with $1 \mathrm{mg} / \mathrm{mL}$ water-soluble DEX. Then, all three groups were air-dried and incubated at $37^{\circ} \mathrm{C}$ in a PBS buffer $(3 \mathrm{~mL})$. Aliquots were then taken from the supernatant on a daily basis over a period of 9 days. The DEX solution was mixed with cupric ions in a $\mathrm{pH} 11$ buffer. Because of the reduction of cupric ions, a cuprous cation was produced and spectrophotometrically detected by incorporating with 2-(4carboxyquinolin-2-yl) quinoline-4-carboxylic acid (Pierce Biotechnology, Rockford, IL). DEX concentrations were quantified by comparing with the standard curve. The accumulated DEX release curve was presented as the result.

\section{Contact angle measurements}

To confirm the adsorption of K1 on the glass slides, the contact angles of $100 \mu \mathrm{L}$ water droplets were measured before and after coating with a $\mathrm{K} 1$ solution $(100 \mu \mathrm{g} / \mathrm{mL})$ using a static contact angle meter (KRÜSS, FM40, Hamburg, Germany). An accurate auto pipette was used to ensure that the volume of the sample $(20 \mu \mathrm{L})$ remained constant across glass slides. The contact angles were measured approximately 30 seconds after the droplets were placed on the surfaces.

\section{Bioactivity}

Osteoblasts (bone-forming cells, ATCC, CRL-11372 population numbers $<9$ ) were seeded at 1,000 cells $/ \mathrm{cm}^{2}$ in well plates and were cultured in Dulbecco's modified Eagle medium (DMEM) medium (Gibco/BRL, Grand Island, NY) supplemented with $10 \%$ fetal bovine serum (FBS; HyClone, Logan, UT) and 1\% penicillin/streptomycin (P/S; Hyclone, Logan, UT) under standard cell culture conditions $\left(37^{\circ} \mathrm{C}\right.$, humidified, $5 \% \mathrm{CO}_{2} / 95 \%$ air environment). DEX-containing samples $(10 \mu \mathrm{L})$ (see controlled release experiments) and freshly prepared and sterilized DEX $\left(10^{-8} \mathrm{M}\right)$ were added to the wells along with the cell culture medium. After the prescribed time period, non-adherent cells were removed by washing twice with phosphate buffered saline (PBS). At the end of 1, 3 and 5 days, osteoblasts were fixed with $10 \%$ normal buffered formalin (NBF; Fisher Scientific, 
Table I 'H NMR spectroscopy of DEX and RNT-encapsulated DEX

\begin{tabular}{|c|c|c|c|c|c|c|}
\hline DEX peaks & $\mathbf{I}$ & 2 & 3 & 4 & 5 & 6 \\
\hline Chemical shift (ppm) & 7.39 & 6.28 & 6.07 & 4.59 & 4.24 & 3.08 \\
\hline ' $\mathrm{H}$ integration of DEX before encapsulation & 1.43 & 1.29 & 1.30 & 1.45 & 2.89 & 1.47 \\
\hline 'H integration of DEX after encapsulation & 0.98 & 0.92 & 0.95 & 0.97 & 1.95 & 0.96 \\
\hline$\%$ Change & 32 & 29 & 27 & 33 & 33 & 35 \\
\hline
\end{tabular}

Notes: The internal standard (t-BuOH) has a chemical shift of $1.22 \mathrm{ppm}$ and an integration corresponding to 9 protons both before and after encapsulation of DEX. See supporting information section for NMR spectra.

Abbreviations: DEX, dexamethasone; NMR, nuclear magnetic resonance; RNT, rosette nanotubes.

Waltham, MA), stained with 4,6-diamidino-2-phenylindole, dihydrochloride (DAPI; Invitrogen, Carlsbad, CA), and counted at five random fields of view for each well under a fluorescence microscope (Zeiss Axiovert 200M, Peabody).

\section{Statistical analysis}

Data were expressed using the standard error of the mean (SEM). Statistics were performed using a Student's one-tailed $t$-test, with $P<0.05$ considered statistically significant.

\section{Results}

\section{Characterization of Drug-loaded RNT-KI}

NMR and DOSY were used to characterize the incorporation between K1 and DEX. Due to their large molecular weight and long relaxation time, $\mathrm{K} 1$ and $\mathrm{K} 1$-encapsulated DEX were not detectable by ${ }^{1} \mathrm{H}$ NMR spectroscopy. Therefore, the percentage of drug loading could only be inferred from differences in peak heights of the drug relative to an internal standard $(t-\mathrm{BuOH})$. As shown in Table 1 , at the DEX: $\mathrm{K}-\mathrm{G} \wedge \mathrm{C}$ molar ratio of $5: 3$, approximately $32 \%$ of DEX was captured by $\mathrm{K} 1$. In agreement with this result, a significant change in the diffusion coefficient of DEX was observed in the DOSY spectra (Table 2).

Tapping mode atomic force microscopy (AFM) showed significantly different height profiles for K1 before and after incorporation of DEX (Figure 2). In addition, the UV-Vis spectra recorded in methanol showed a significant hypochromic effect at $280 \mathrm{~nm}$ and a hyperchromic effect around $240 \mathrm{~nm}$. In water, on the other hand, a dramatic hyperchromic effect was observed around 280 and $240 \mathrm{~nm}$ suggesting that DEX significantly altered the stacking interaction in K1 (Figure 3).

\section{Drug loading and release experiments}

For drug loading experiments, K1 samples with/without incorporation of DEX were prepared on glass slides. To confirm that $\mathrm{K} 1$ adhered to the glass slide surfaces, contact angle measurements were carried out. A notable decrease in contact angles (from $54.1 \pm 2.1$ to $43.9 \pm 3.5$ ) for the K1-coated slides was measured confirming that the hydrophilicity of the glass slide surface increased upon K1 adsorption.

To further test drug loading ability and release kinetics of $\mathrm{K} 1$, a release experiment was conducted in a physiological environment: specifically, in water buffer and at $37^{\circ} \mathrm{C}$ incubation. Two approaches were used to incorporate DEX with K1. The first approach consisted of physically adsorbing $\mathrm{K} 1$ onto a glass slide and then dipping the slide in a water-soluble DEX solution. The second approach consisted of assembling $\mathrm{K} 1$ in the water-soluble DEX solution and then adsorbing the complex on a glass slide. Results showed that $\mathrm{K} 1$ in the first approach retained approximately $24.8 \%$ more DEX than the control sample (uncoated glass slide dipped in water-soluble DEX solution) and released DEX up to 6 days. Using the second approach, K1 incorporated approximately $42 \%$ more DEX than the control sample (Figure 4).

\section{Drug bioactivity tests}

After establishing the ability of K1 to incorporate and slowly release drugs, one important concern is whether the released drugs are still bioactive. To test the bioactivity of the drug, commercially available fresh DEX, along with released DEX from glass slides and from K1 were used as additives separately for osteoblast culture. As shown in Figure 5,

Table 2 DOSY NMR of DEX only and RNT-encapsulated DEX

\begin{tabular}{llllllll}
\hline DEX peaks & $\mathbf{I}$ & $\mathbf{2}$ & $\mathbf{3}$ & $\mathbf{4}$ & $\mathbf{5}$ & $\mathbf{6}$ & Average \\
\hline Chemical shift (ppm) & 7.39 & 6.28 & 6.07 & 4.59 & 4.24 & 3.08 & - \\
$\begin{array}{l}\text { Diffusion Coefficient } \\
\left(\mathrm{m}^{2} / \mathrm{s}\right) \text { of DEX before }\end{array}$ & 5.6 & 5.8 & 5.8 & 5.8 & 6 & 5.8 & 5.8 \\
encapsulation & & & & & & & \\
$\begin{array}{l}\text { Diffusion Coefficient } \\
\left(\mathrm{m}^{2} / \mathrm{s}\right) \text { of DEX after }\end{array}$ & 5 & 5 & 5 & 5.2 & 5.4 & 5 & 5.1 \\
encapsulation & & & & & & &
\end{tabular}

Notes: The internal standard (t-BuOH) has a chemical shift of $1.22 \mathrm{ppm}$ and an integration of corresponding to 9 protons both before and after encapsulation of DEX. See supporting information section for NMR spectra.

Abbreviations: DEX, dexamethasone; NMR, nuclear magnetic resonance; RNT, rosette nanotubes; DOSY NMR, diffusion ordered nuclear magnetic resonance spectroscopy. 

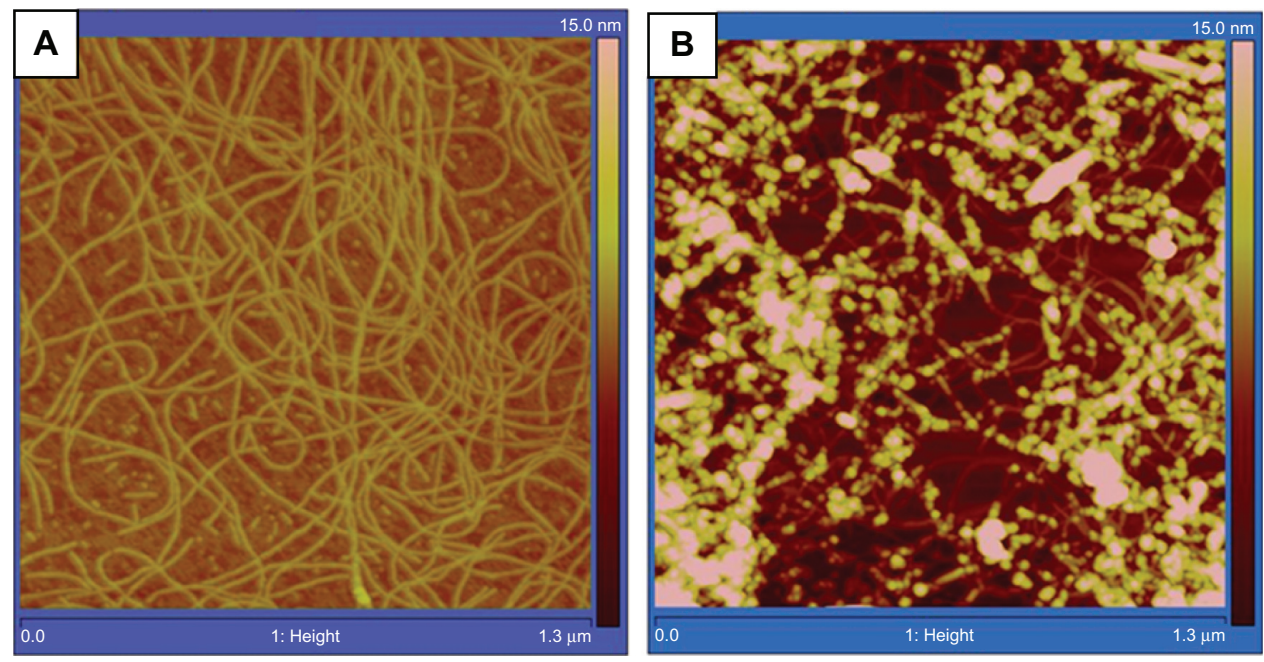

Figure 2 Tapping mode AFM height profiles of (A) RNTs and (B) RNT-encapsulated DEX. Average heights measured for RNTs and RNT-DEX complexes were $2.91 \pm 0.95 \mathrm{~nm}$ and $7.04 \pm 0.33 \mathrm{~nm}$, respectively.

Abbreviations: AFM, Atomic force microscopy; RNT, rosette nanotubes; DEX, dexamethasone.

osteoblast densities from the three groups with DEX were higher relative to the negative controls (without DEX). Importantly, DEX released from K1 increased osteoblast density the most.

\section{Discussion}

Traditional drug delivery systems involve chemical or physical modification to bind with specific drugs for their controlled release. Chemical modification aims to establish chemical bonds (such as covalent bonds) between the drug and the carriers. Physical adsorption employs simple procedures, but gives low drug loading and fast release under physiological conditions, thus resulting in limited cell-drug interactions. ${ }^{24,25}$ However, RNTs have unique design features

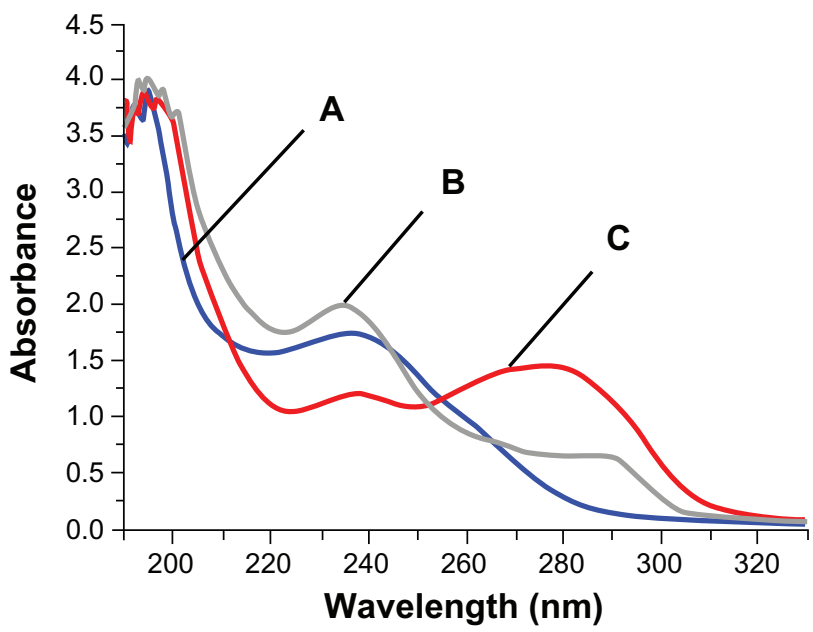

that make them attractive for drug delivery purposes. Their hydrophobic core resulting from base stacking and selfassembly attracts and entraps small hydrophobic molecules behind a hydrophilic and biocompatible outer surface. Entrapped, water insoluble drugs can thus be delivered under physiological conditions to bone cells. ${ }^{11,12}$

In this study, DEX was chosen as a model drug because of its wide use in orthopedic applications since it is an antiinflammatory agent and can control stem cell differentiation into osteoblasts. ${ }^{26,27}$ DOSY NMR and AFM suggested the incorporation of DEX in K1. Moreover, since UV-Vis spectroscopy showed a significant hyperchromic effect upon DEX encapsulation by $\mathrm{K} 1$ in water, any change in K1's UV-Vis spectrum may be associated with the formation of a

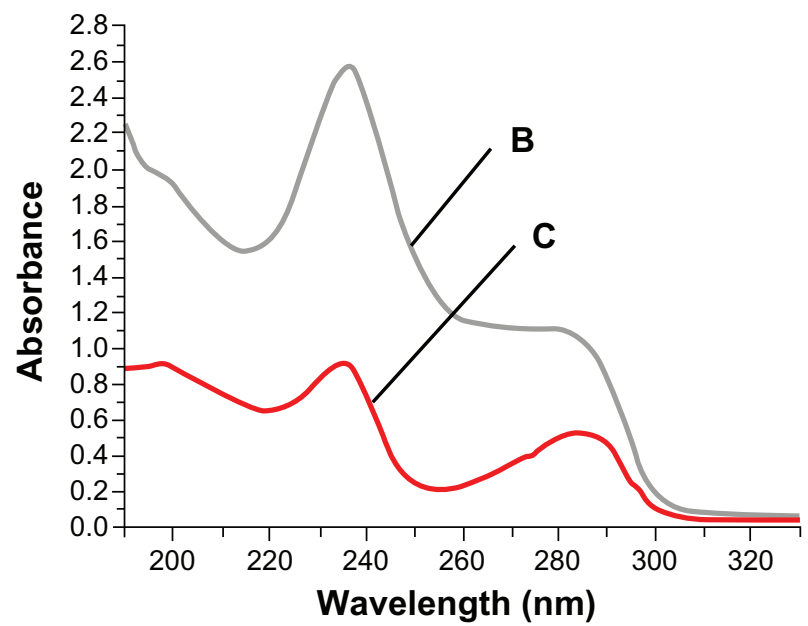

Figure 3 Comparison of UV-Vis absorbance curves in methanol (Left) and water (Right). A) $0.005 \mathrm{mg} / \mathrm{mL}$ DEX, B) $0.025 \mathrm{mg} / \mathrm{mL}$ DEX-RNTs and C) $0.025 \mathrm{mg} / \mathrm{mL}$ RNTs. There is no UV absorbance curve of DEX only in water, because DEX precipitates in water. Abbreviations: RNT, rosette nanotubes; DEX, dexamethasone. 


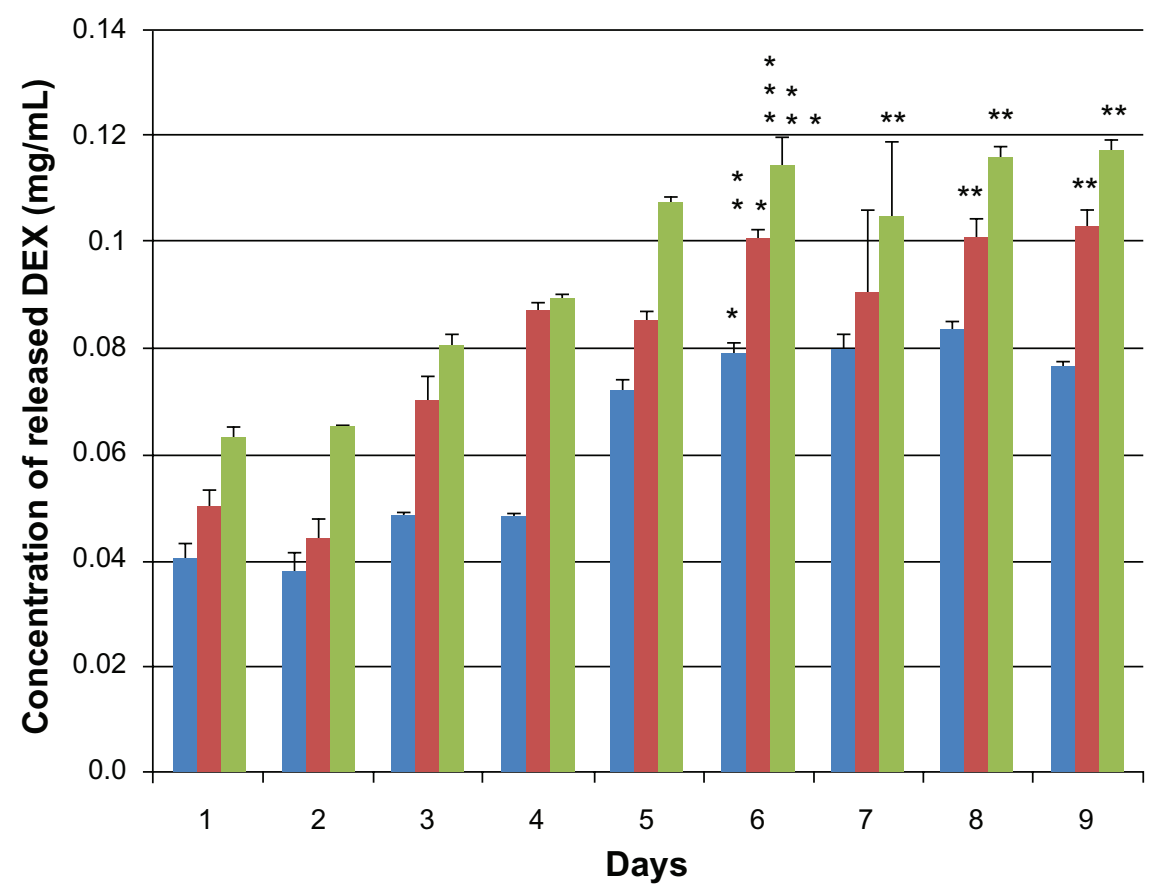

Group a: DEX released from glass slides

Group b: DEX released from RNT coated glass slides

Group c: DEX released from $\mathrm{DEX}-\mathrm{K} 1$ on glass

Figure 4 Accumulated DEX release curves up to 9 days.

Note: Data are mean \pm SEM $(n=12)$. *P $<0.05$ compared to DEX concentrations at day I to $5 ; * * P<0.05$ compared to group (a) at respective days; $* * * P<0.05$ compared to group (a) and group (b) at the respective day.

Abbreviations: DEX, dexamethasone; SEM, standard error in the mean.

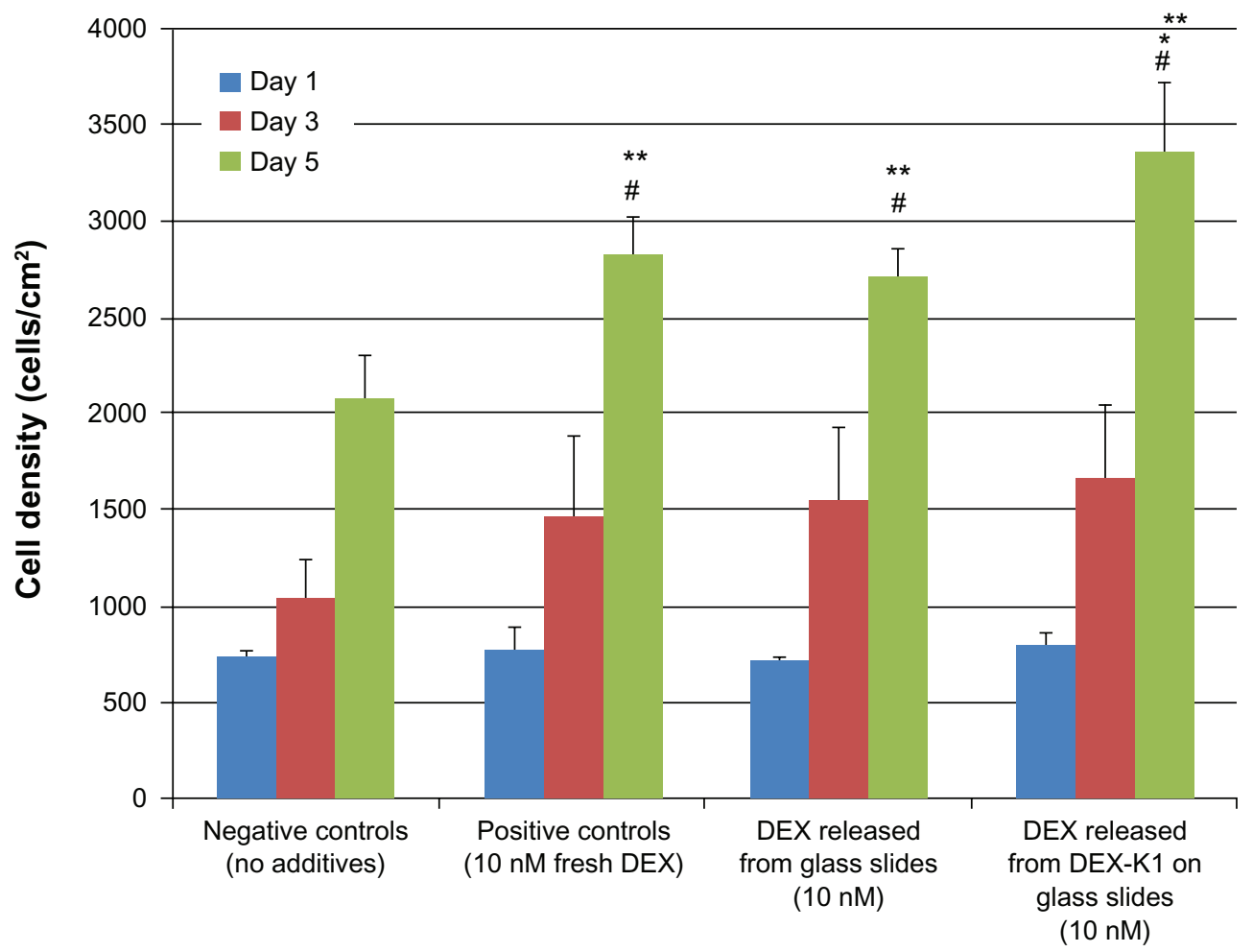

Figure 5 Osteoblast density cultured with released DEX.

Notes: Data are mean \pm SEM $(n=9)$. $* P<0.05$ compared to both controls and DEX released from glass slides at day 5 ; $* * P<0.05$ compared to negative controls (no additives) at day 5 ; ${ }^{\#}<0.05$ compared to day $I$ and 3 results on respective samples.

Abbreviations: DEX, dexamethasone; SEM, standard error in the mean. 
stable DEX-K1 complex, possibly via intercalation/stacking interactions.

To further understand how DEX is incorporated into K1 and to determine the DEX loading and release behavior, the DEX-K1 complex was prepared using two different methods: 1) adsorbing DEX onto K1 coated glass slides; and 2) assembling K1 with DEX solution. Results showed that the second method led to a larger amount of DEX loading, and a prolonged release under physiological conditions $\left(37^{\circ} \mathrm{C}\right.$ in aqueous buffer). These results support the idea that DEX is entrapped inside the hydrophobic core of $\mathrm{K} 1$.

Equally important is the biological activity of the released DEX. Since DEX has been reported to increase cell density, ${ }^{22,28}$ we investigated osteoblast cell cultures with no additives (negative controls), commercially available water-soluble DEX (positive controls), released DEX from uncoated glass slides, and released DEX from the DEX-K1-coated glass slides. Higher osteoblast densities from the three groups with DEX were observed compared to the negative control (without DEX). In addition, DEXK1-coated glass slides not only exerted the same biological effect as the drug released from DEX-coated glass slides, but also promoted higher osteoblast density, in agreement with earlier reports. ${ }^{11,12,17,18,29}$

In summary, these results suggest that DEX was incorporated into $\mathrm{K} 1$ in the process of self-assembly by hydrophobic and base stacking interactions. Thus, for orthopedic applications, RNTs not only enhance osteoblast adhesion and subsequent functions (such as calcium deposition) as previously shown, ${ }^{11,12,20}$ but they can also incorporate water-insoluble drugs (such as DEX) and release them in physiological media over an extended period of time.

\section{Conclusions}

We have demonstrated that RNTs have unique chemical and physical properties that make them particularly attractive for drug delivery applications. For instance, they can be modified: a) chemically with various peptides to tailor them for specific tissue-engineering applications; b) physically, by drug-entrapment within their core; and c) structurally, by synthetically altering their dimensions (length, diameter) to accommodate a broad range of therapeutic modalities.

The incorporation of DEX into $\mathrm{K} 1$ was established by ${ }^{1} \mathrm{H}-\mathrm{NMR}$ spectroscopy, DOSY NMR, UV-Vis spectroscopy, AFM, and in vitro osteoblast cultures. This study demonstrated that K1, a member of the RNT family, can encapsulate DEX via a simple self-assembly process, and release it under physiological conditions, over an extended period of time spanning 9 days. The released drug enhanced the established biological activity of $\mathrm{K} 1$. We envision, as a result, several applications in drug delivery for the RNT platform.

\section{Acknowledgments}

This work was supported by the National Research Council of Canada, the Natural Science and Engineering Research Council of Canada, and the Hermann Foundation. The authors also thank Dr Jae-Young Cho for assistance with AFM measurements.

\section{Disclosure}

The authors report no conflicts of interest in this work.

\section{References}

1. Charnley J. Anchorage of the femoral head prosthesis to the shaft of the femur. J Bone Joint Surg Br. 1960;42-B:28-30.

2. Sato N, Kubo K, Yamada M, et al. Osteoblast mechanoresponses on Ti with different surface topographies. J Dent Res. 2009;88(9):812-816.

3. Shi GA, Ren LF, Wang LZ, Lin HS, Wang SB, Tong YQ. $\mathrm{H}_{2} \mathrm{O}_{2} / \mathrm{HCl}$ and heat-treated Ti-6Al-4V stimulates pre-osteoblast proliferation and differentiation. Oral Surg Oral Med Oral Pathol Oral Radiol Endod. 2009;108(3):368-375.

4. Kuo YC, Yeh CF, Yang JT. Differentiation of bone marrow stromal cells in poly(lactide-co-glycolide)/chitosan scaffolds. Biomaterials. 2009;30(34):6604-6613.

5. Vidigal GM Jr, Groisman M, Gregório LH, Soares Gde A. Osseointegration of titanium alloy and HA-coated implants in healthy and 1 animals: a histomorphometric study. Clin Oral Implants Res. 2009;20(11):1272-1277.

6. Balasundaram G, Webster TJ. Nanotechnology and biomaterials for orthopedic medical applications. Nanomedicine (Lond). 2006;1(2): 169-176.

7. Balasundaram G, Webster TJ. An overview of nano-polymers for orthopedic applications. Macromol Biosci. 2007;7(5):635-642.

8. Bozic KJ, Kurtz SM, Lau E, Ong K, Vail TP, Berry DJ. The epidemiology of revision total hip arthroplasty in the United States. J Bone Joint Surg (Am). 2009;91:128-133.

9. Chan CK, Kumar TS, Liao S, Murugan R, Ngiam M, Ramakrishnan S. Biomimetic nanocomposites for bone graft applications. Nanomedicine (Lond). 2006;1(2):177-188.

10. Choksi AN, Poonawalla T, Wilkerson MG. Nanoparticles: a closer look at their dermal effects. J Drugs Dermatol. 2010;9(5):475-481.

11. Chun AL, Moralez JG, Fenniri H, Webster TJ. Helical rosette nanotubes: a biomimetic coating for orthopedics? Biomaterials. 2005;26(35): 7304-7309.

12. Chun AL, Moralez JG, Fenniri H, Webster TJ. Helical rosette nanotubes: A more effective orthopaedic implant material. Nanotechnology. 2004;15:s234-s239.

13. Fenniri H, Mathivanan P, Vidale KL, et al. Helical rosette nanotubes: design, self-assembly and characterization. J Am Chem Soc. 2001;123(16):3854-3855.

14. Journeay WS, Suri SS, Moralez JG, Fenniri H, Singh B. Rosette nanotubes show low acute pulmonary toxicity in vivo. Int $J$ Nanomedicine. 2008;3(3):373-383.

15. Journeay WS, Suri SS, Moralez JG, Fenniri H, Singh B. Low inflammatory activation by self-assembling Rosette nanotubes in human Calu-3 pulmonary epithelial cells. Small. 2008;4(6):817-823. 
16. Suri SA, Rakotondradany F, Myles AJ, Fenniri H, Singh B. The role of RGD-tagged rosette nanotubes in the induction of inflammation and apoptosis in human adenocarcinoma cells through the p38 MAPK pathway. Biomaterials. 2009;30(17):3084-3090.

17. Zhang L, Chen Y, Rodriguez J, Fenniri H, Webster TJ. Biomimetic helical rosette nanotubes and nanocrystalline hydroxyapatite coatings on titanium for improving orthopedic implants. Int $J$ Nanomedicine. 2008;3(3):323-333.

18. Zhang L, Rakotondradany F, Myles AJ, Fenniri H, Webster TJ. Arginineglycine-aspartic acid modified rosette nanotube-hydrogel composites for bone tissue engineering. Biomaterials. 2009;30(7):1309-1320.

19. Song S, Chen Y, Yan Z, Fenniri H, Webster TJ. Self-assembled rosette nanotubes for incorporating hydrophobic drugs in physiological environments. Int J Nanomedicine. 2011;10(6):101-107.

20. Guzmán-Morales J, El-Gabalawy H, Pham MH, et al. Effect of chitosan particles and dexamethasone on human bone marrow stromal cell osteogenesis and angiogenic factor secretion. Bone. 2009;45(4):617-626.

21. Beule AG, Steinmeier E, Kaftan H, et al. Effects of a dexamethasonereleasing stent on osteoneogenesis in a rabbit model. Am J Rhinol Allergy. 2009;23(4):433-436.

22. Yang L, Tao T, Wang X, et al. Effects of dexamethasone on proliferation, differentiation and apoptosis of adult human osteoblasts in vitro. Chin Med J (Engl). 2003;116(9):1357-1360.
23. Grigoriadis AE, Heersche JN, Aubin JE. Differentiation of muscle, fat, cartilage, and bone from progenitor cells present in a bone-derived clonal cell population: effect of dexamethasone. J Cell Biol. 1988; 106(6):2139-2151.

24. Yang L, Webster TJ. Nanotechnology controlled drug delivery for treating bone diseases. Expert Opin Drug Deliv. 2009;6(8):851-864.

25. Gindy ME, Prud'homme RK. Multifunctional nanoparticles for imaging, delivery and targeting in cancer therapy. Expert Opin Drug Deliv. 2009;6(8):865-878.

26. Short DJ, El Masry WS, Jones PW. High dose methylprednisolone in the management of acute spinal cord injury - a systematic review from a clinical perspective. Spinal Cord. 2000;38(5):273-286.

27. Morimoto D, Kuroda S, Kizawa T, et al. Equivalent osteoblastic differentiation function of human mesenchymal stem cells from rheumatoid arthritis in comparison with osteoarthritis. Rheumatology (Oxford). 2009;48(6):643-649.

28. Guerriero V, Florini JR. Dexamethasone effects on myoblast proliferation and differentiation. Endocrinology. 1980;106(4):1198-1202.

29. Chen Y, Bilgen B, Pareta RA, et al. Self-assembled rosette nanotube/ hydrogel composites for cartilage tissue engineering. Tissue Eng Part C Methods. 2010;16(6):1233-1243. 


\section{Supplementary figures}

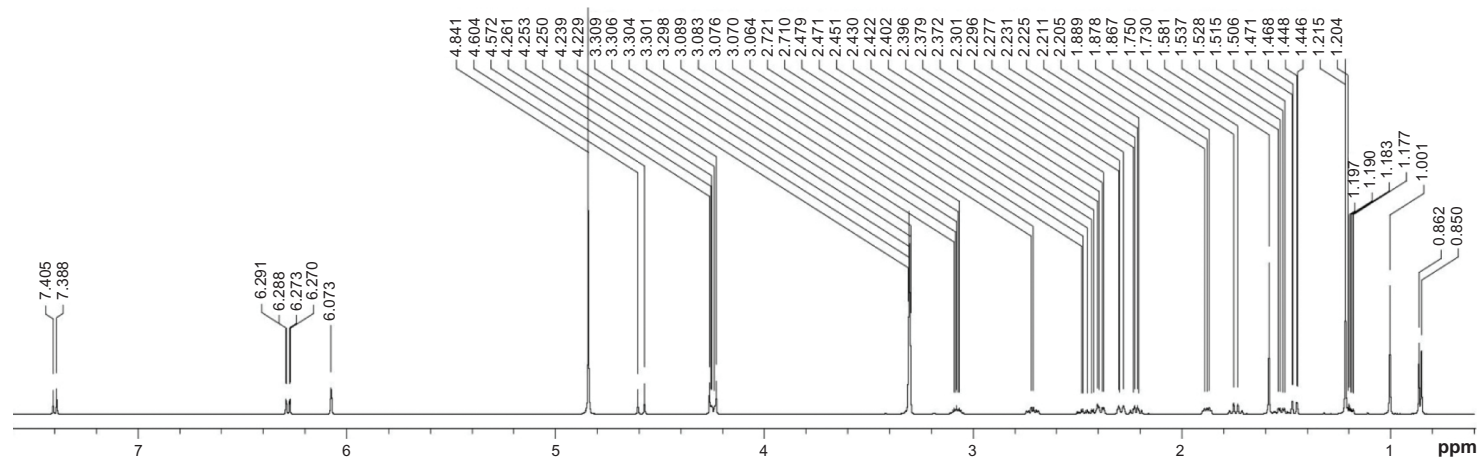

Figure SI 'H spectrum of DEX control (before incorporating with RNTs).

Abbreviations: RNT, rosette nanotubes; DEX, dexamethasone.

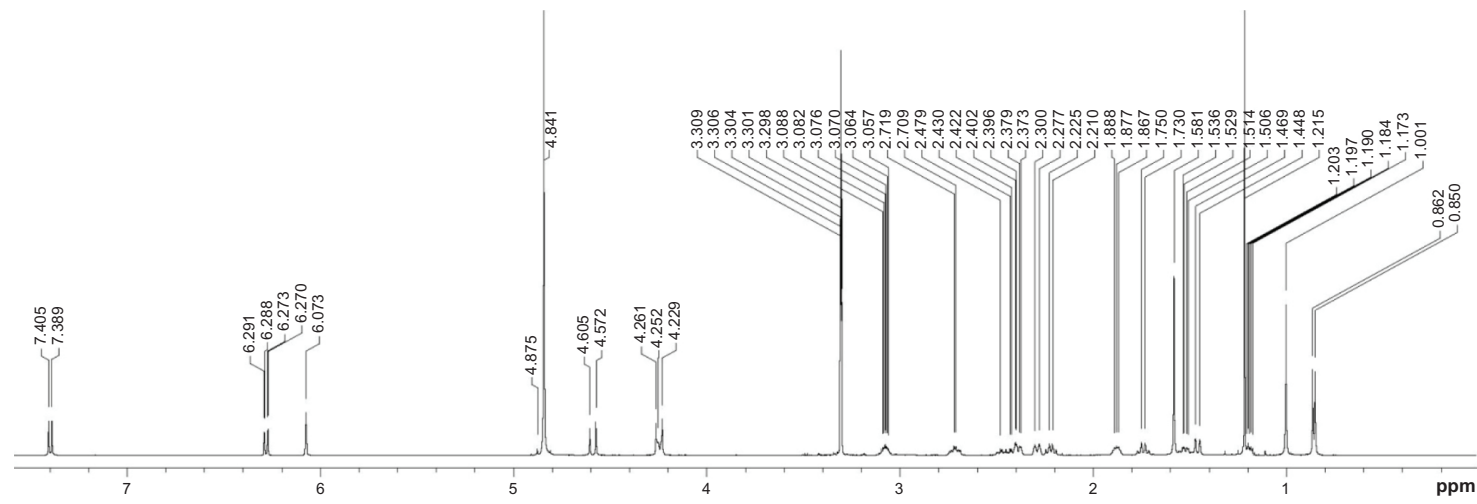

Figure S2 'H spectrum of DEX/RNT composites (after incorporating with RNTs)

Abbreviations: RNT, rosette nanotubes; DEX, dexamethasone.

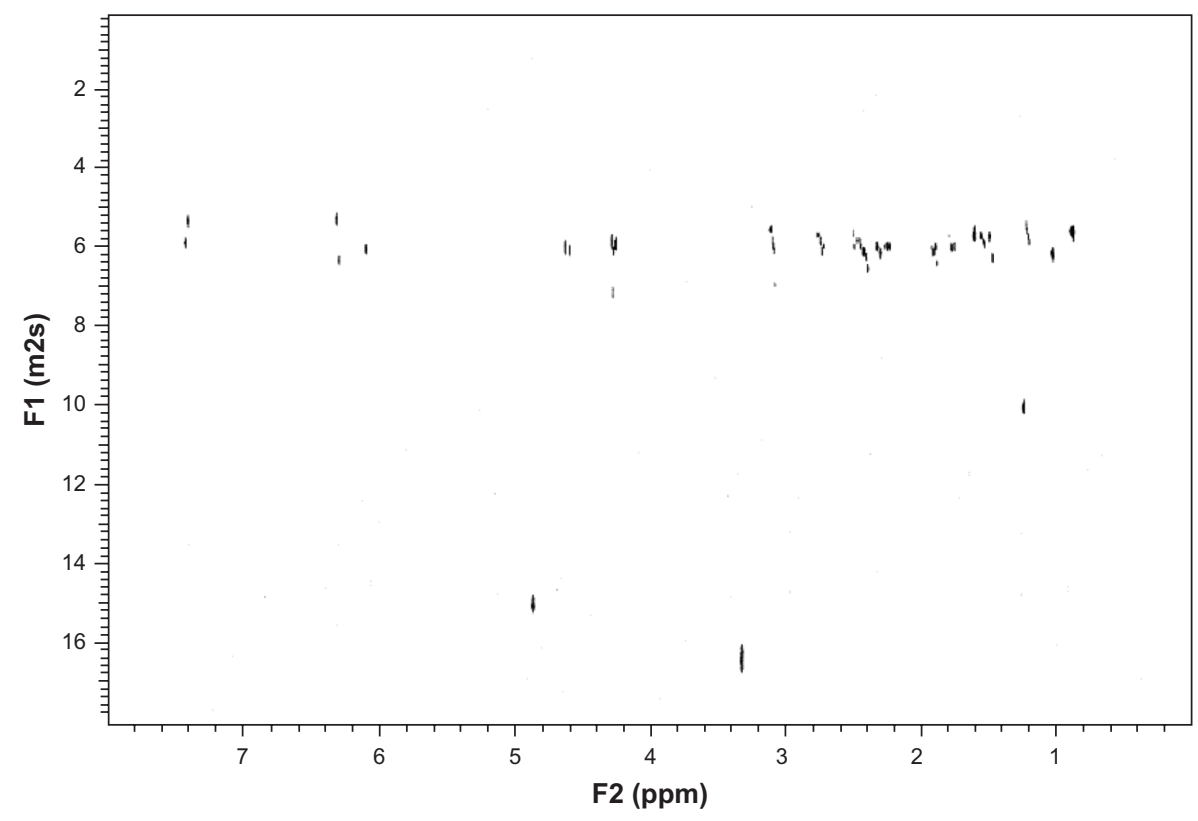

Figure S3 DOSY of DEX (before incorporating with RNTs).

Abbreviations: RNT, rosette nanotubes; DEX, dexamethasone; DOSY, diffusion ordered nuclear magnetic resonance spectroscopy. 


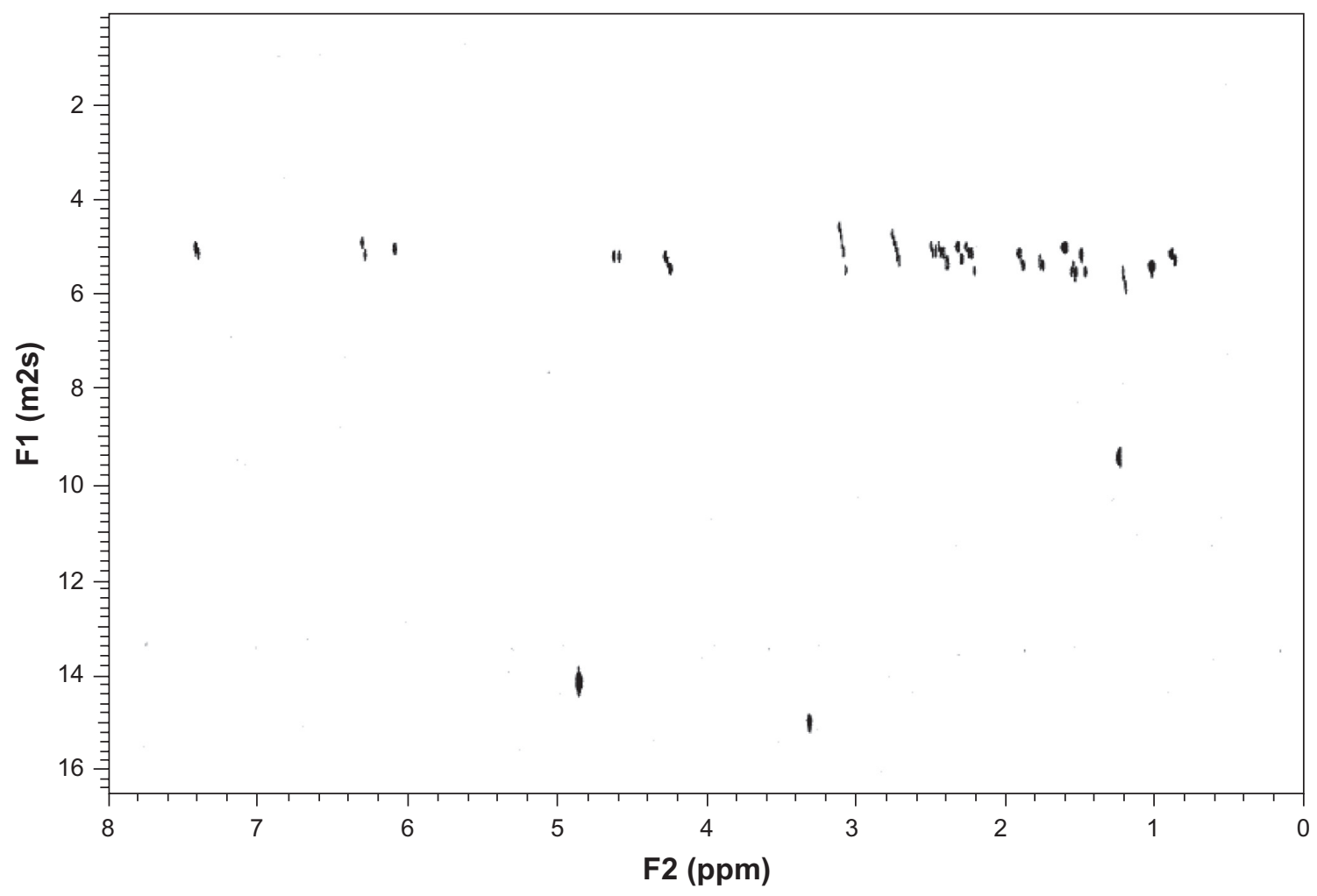

Figure S4 DOSY of DEX/RNT (after incorporating with RNTs).

Abbreviations: RNT, rosette nanotubes; DEX, dexamethasone; DOSY, diffusion ordered nuclear magnetic resonance spectroscopy.

\section{Publish your work in this journal}

The International Journal of Nanomedicine is an international, peerreviewed journal focusing on the application of nanotechnology in diagnostics, therapeutics, and drug delivery systems throughout the biomedical field. This journal is indexed on PubMed Central, MedLine, CAS, SciSearch ${ }^{\circledR}$, Current Contents ${ }^{\circledR} /$ Clinical Medicine,
Journal Citation Reports/Science Edition, EMBase, Scopus and the Elsevier Bibliographic databases. The manuscript management system is completely online and includes a very quick and fair peer-review system, which is all easy to use. Visit http://www.dovepress.com/ testimonials.php to read real quotes from published authors. 